\title{
Efficacy of a New Tonic Containing Urea, Lactate, Polidocanol, and Glycyrrhiza inflata Root Extract in the Treatment of a Dry, Itchy, and Subclinically Inflamed Scalp
}

\author{
Dorothea Schweiger Caroline Baufeld Philip Drescher Bernhardt Oltrogge \\ Simone Höpfner Aylin Mess Juliane Lüttke Frank Rippke Alexander Filbry \\ Heiner Max
}

Research and Development, Beiersdorf AG, Hamburg, Germany

\section{Key Words \\ Licochalcone A · Urea · Lactate · Polidocanol · Scalp · \\ Xerosis $\cdot$ Itch $\cdot$ Microinflammation $\cdot$ Leave-on tonic}

\begin{abstract}
Background/Aims: Dry, itchy and inflamed scalp conditions are common and often associated with diseases such as atopic dermatitis or psoriasis. To improve these symptoms, we investigated the efficacy of a new tonic containing the active ingredients urea, lactate, polidocanol, and Glycyrrhiza inflata root extract, containing licochalcone A. Study Design/Methods: 30 subjects with dry and itchy scalp conditions underwent a randomized half-head study for 4 weeks, applying the leave-on tonic three times a week on one side of the scalp. Tonic effects on skin hydration, itching, lipids, microinflammation, and substantivity of tonic compounds were determined using corneometry, middleinfrared spectroscopy, direct analysis in real-time mass spectrometry, and enzyme-linked immunosorbent assay. Volunteers performed a self-assessment; changes in scalp condition were documented by in vivo microscopy. $\boldsymbol{R e}$ sults: After tonic treatment, scalp moisture was significantly increased, whereas scalp itching and tautness were significantly reduced. Results also demonstrated a high sub-
\end{abstract}

\begin{tabular}{ll}
\hline KARGER & $\begin{array}{l}\text { ( } 2013 \text { S. Karger AG, Basel } \\
1660-5527 / 13 / 0262-0108 \$ 38.00 / 0 \quad \text { Karger }\end{array}$ \\
E-Mail karger@karger.com & $\begin{array}{l}\text { This is an Open Access article licensed under the terms } \\
\text { www.karger.com/spp }\end{array}$ \\
& $\begin{array}{l}\text { of the Creative Commons Attribution-NonCommercial- } \\
\text { NoDerivs 3.0 License (www.karger.com/OA-license), appli- } \\
\text { cable to the online version of the article only. Distribution } \\
\text { for non-commercial purposes only. }\end{array}$
\end{tabular}

stantivity of urea and lactate on the scalp, an increase in triglyceride, and a decrease in free fatty acid levels. The amount of total lipids was unchanged. Analyses of scalp wash-ups verified a significant reduction in important proinflammatory markers. Conclusion: Due to the actives urea, lactate, polidocanol, and the anti-inflammatory licochalcone $A$, the new scalp tonic exhibited excellent performance in alleviating scalp dryness, itching, microinflammation, and in normalizing disturbances of scalp lipids.

Copyright $\odot 2013$ S. Karger AG, Base

\section{Introduction}

Human scalp represents a unique body area characterized by high hair density, as well as high density of sebaceous and sweat glands. These particular conditions may result in the scalp developing certain disorders manifested in symptoms such as pruritus, dryness/oiliness, and tautness [1]. According to a survey including a representative sample of the French population, 25\% of the study population suffered from scalp itch and $24 \%$ reported dry scalp skin [2].

Xerotic scalp conditions can be caused by intrinsic and extrinsic factors such as climate, chemicals, drugs, micro- 
organisms, cosmetics, as well as genetics, ageing, and stress $[3,4]$. One of the most common skin conditions linked to dry skin is itch. Pruritus is commonly associated with skin diseases such as seborrheic dermatitis, skin xerosis, atopic dermatitis, as well as psoriasis. However, scalp pruritus can often be present without any visible skin lesions or being associated with a particular disease [5].

Treatment options for dry skin involve improvement in skin hydration by supplementation with natural moisturizing factors (NMFs), the osmotically active molecules within the corneocytes. Natural moisturizing factors such as the humectants, lactate and urea, are prevalent in the stratum corneum (SC) [6]; many dry-skin-related skin conditions such as atopic dermatitis or psoriasis have been linked to reduced NMF levels [3, 7].

To control pruritus in xerotic skin, a topical formulation containing the non-ionic surfactant polidocanol has been successfully applied in combination with urea [8]. In addition, polidocanol has been shown to exhibit local anesthetic effects [9].

Aside from the lack of hydration, a dry and/or itchy scalp e.g. in atopic dermatitis is often associated with an impaired barrier function, alterations in SC and hair lipids, and focal (micro)inflammatory processes $[10,11]$. In this context, the flavonoid licochalcone A, a component isolated from the root of the licorice species Glycyrrhiza inflata, came to our attention. In China, licorice traditionally represents one of the most frequently utilized drugs [12]. Licochalcone A exhibits anti-inflammatory and antimicrobial activity [12-14] and has been shown to suppress NF- $\kappa \mathrm{B}$-regulated gene products and inhibit tumor necrosis factor $\alpha$ (TNF- $\alpha$ )-induced inflammation [15]. With respect to human skin, licochalcone A exhibited anti-inflammatory activity in vitro and in vivo [16, 17]. Topical treatments with formulations consisting of Glycyrrhiza inflata root extract, which contained licochalcone A, demonstrated beneficial effects on human skin in models of skin irritation [17] and in patients with erythematotelangiectatic rosacea and red facial skin [18]. Also, patients suffering from atopic dermatitis benefited from treatment with licochalcone A [19].

In this study, we focused on the effects of a leave-on tonic comprising urea, lactate, polidocanol, and Glycyrrhiza inflata root extract (containing licochalcone A) in the treatment of dry and/or itchy scalp. Regular use of the tonic resulted in a significant improvement in scalp condition. Tonic application increased skin hydration, decreased pruritus, normalized lipid levels in the SC, and reduced levels of pro-inflammatory markers.

Leave-on Tonic for Treating Xerotic, Itchy and Subclinically Inflamed Scalp

\section{Materials and Methods}

\section{Test Tonic}

The topical aqueous leave-on tonic (EUCERIN ${ }^{\circledR}$-DermoCapillaire; Beiersdorf AG, Hamburg, Germany) comprised Glycyrrhiza inflata root extract which contained licochalcone A as active ingredient [17]. Further active ingredients were urea, polidocanol, and lactate (INCI: aqua, urea, methylpropanediol, sodium lactate, laureth-9, Glycyrrhiza inflata root extract, glycerin, lactic acid, arginine $\mathrm{HCl}$, PEG-40 hydrogenated castor oil, cetrimonium chloride, phenoxyethanol).

\section{In vivo Study}

Determination of Tonic Effects on the Scalp

Suitable volunteers displaying a dry and itchy scalp were selected in a prescreening from a pool of subjects known to suffer from dry skin. These volunteers are registered at our test center, had already participated in previous studies and showed no indication of bacterial/fungal infections of the scalp or other scalp diseases.

The first inclusion criterion for the study was the status of scalp hydration, which was assessed by measuring skin conductivity. To be eligible for inclusion in the study, volunteers had to display a value $<20 \mu \mathrm{S}$. As a second criterion, volunteers had to show a scalpitching and/or tautness score $\geq 2$, as determined by volunteer selfassessment. Thirdly, scalp oiliness of $<2.5$ had to be present, as established by expert visual assessment of the scalp surface. The various symptoms, scalp itching, tautness, and oiliness were determined based on the following assessment scale (scale 0-5): $0=$ no characteristic symptom; 1 = weak, even visible/perceivable symptom; 2 = mild symptom; 3 = moderate symptom; 4 = strong symptom; 5 = very strong (severe) symptom. Thirty out of 153 volunteers (13 male and 17 female, $26-73$ years) met the above criteria and were enrolled in the study.

In this in vivo study, the recommendations of the current version of the Declaration of Helsinki and the guideline of the International Conference on Harmonization Good Clinical Practice (ICH GCP) were observed as applicable to a non-drug study. All volunteers provided written, informed consent. The study was conducted based on a randomized, investigator-blinded, split-scalp design and carried out under dermatological supervision.

To standardize scalp condition prior to the study start, all volunteers took part in a 2-week conditioning phase. During this period, a commercially available standard shampoo without any actives was supplied by Beiersdorf AG for use at home. The shampoo was required to be applied as practiced in everyday use, but at least 2 to 3 times a week. During the conditioning phase and also throughout the study, the extra use of rinse-off or leaveon hair care products, unscheduled washing of the hair performed by the volunteer, hair dyeing or coloration, excessive hair styling, and chemical/physical hair treatment were prohibited. The last hair wash, use of styling products such as wax, gel or foam, as well as visits to saunas, tanning salons, swimming pools, and taking part in intensive sports associated with strong perspiration were not allowed $24 \mathrm{~h}$ prior to each scheduled measurement.

In the course of the 4-week treatment period, the volunteers' scalp was treated only in the hair studio at the Beiersdorf test center. The standard shampoo and the test tonic were applied by 
trained, supervised experts 3 times a week according to written instructions. Test sites were the right and the left sides of the scalp. After washing, one side was treated with the test tonic and the other side was left untreated. Positioning of the treatment locations was permutated from volunteer to volunteer by means of a permutation scheme. Depending on individual volume, quantity and thickness of hair, an amount of approximately $1.5-2.5 \mathrm{~g}$ tonic was used on the test site. Approximately $0.5 \mathrm{~cm}$ of the middle head area was not treated to avoid cross-contamination of test substances. Self-evidently, in this middle head area no measurements were performed or samples taken.

Forty-eight hours prior to measuring and sampling, the scalp was washed, but no application of the test tonic was performed. Analyses were performed after acclimatization of the volunteers for at least $30 \mathrm{~min}$ under standard atmospheric conditions (21.5 \pm $1.0^{\circ} \mathrm{C}$ and $45 \pm 5 \%$ relative humidity).

The following biophysical and optical methods were used to assess tonic effects on scalp parameters:

Determination of Scalp Hydration

The degree of scalp hydration correlates with the inductive conductivity of the skin. To determine scalp hydration, corneometry using the DermaLab ${ }^{\circledR}$ moisture 8-pin module (Cortex Technology, Hadsund, Denmark) was performed at baseline $\left(\mathrm{t}_{0}\right)$ and after $2\left(\mathrm{t}_{1}\right)$ and $4\left(\mathrm{t}_{2}\right)$ weeks of treatment to assess the potential of the test tonic to increase scalp moisturization. For this purpose, the hair was parted with the help of a tail comb and secured using hairclips. Subsequently, 8 measurements were performed on both the treated and the untreated scalp side.

Also, Fourier-transformed middle-infrared spectroscopy (FTMIR; IFS-55 Equinox; Bruker, Bremen, Germany) was utilized to determine changes in scalp hydration by analyzing the amide band ratio in the corresponding spectra at $t_{0}, t_{1}$ and $t_{2}$. To record a spectrum, the volunteer's hair was parted as described above; the volunteer was asked not to move or talk. For measurements, the $\mathrm{N}_{2}$-cooled, diamond measuring head was placed vertically and without pressure on the test site. Five measurements consisting of 40 scans were carried out on both the treated and the untreated side. The spectrometer was equipped with a diamond-ATF-fiber probe $(5 \times 40$ scans; Bruker). Analysis of N-H bonds (1,650-1,550 $\mathrm{cm}^{-1}$ ) was performed using the OPUS NT 3.1 software (Bruker) according to the method described by Triebskorn and coworkers [20].

Scalp Substantivity of Urea and Lactate by Using Direct

Analysis in Real-Time Mass Spectrometry Analysis

To investigate the substantivity of the NMFs, urea and lactate, following tonic treatment, direct analysis in real-time mass spectrometry (DART-MS) was utilized. DART-MS analyses were performed using a DART ion source (IonSense, Inc., Saugus, Mass., USA) coupled to a JEOL AccuTOF orthogonal acceleration singlereflectron time-of-flight (TOF) mass spectrometer (JEOL GmbH, Eching, Germany) [21].

At baseline, swabs were obtained from both sides of the untreated scalp using small glass rods, so-called DIP-it samplers (IonSense, Inc.). After 2 and 4 weeks of treatment, swabs were again obtained from both test sites. For sample collection, the volunteer's hair was parted as described above; the DIP-it sampler was rubbed 10 times with the enclosed end of the glass capillaries, while applying slight, constant pressure. The adherent skin surface material was directly analyzed using DART-MS without further sample preparation. Samples were evaluated for urea and lactate content. Analyte ions were identified by elemental composition calculation (monoisotopic masses, software Elemental Composition Estimation, MassCenter Main, version 1.3.0.1000, Jeol GmbH) and verified by analysis of reference substances. The mass spectrometer settings were as follows: peak voltage $600 \mathrm{~V}$, ring lens voltage $5 \mathrm{~V}$, orifice 1 voltage $20 \mathrm{~V}$, orifice 2 voltage $5 \mathrm{~V}$. All spectra were obtained in a mass range of $\mathrm{m} / z 50-1,000$ and with a spectrum recording interval of $0.2 \mathrm{~s}$.

\section{Determination of Free Fatty Acids and Triglycerides Using FT-MIR}

FT-MIR was utilized to investigate the amount of total lipids, triglycerides and free fatty acids at baseline and after 2 and 4 weeks, comparing the tonic-treated and the untreated scalp side. Analysis of C-H bonds $\left(\mathrm{CH}_{2} / \mathrm{CH}_{3}: 2,800-3,000 \mathrm{~cm}^{-1}\right)$, ester bonds (COOR: $\left.1,740 \mathrm{~cm}^{-1}\right)$, and C-O-single bonds $\left(\mathrm{C}-\mathrm{O}(-\mathrm{H}): 1,040 \mathrm{~cm}^{-1}\right)$ was carried out as described above.

Determination of Scalp Cytokine Levels

For the investigation of interleukin $1 \beta$ (IL-1 $\beta$ ), interleukin 1 receptor antagonist (IL-1ra), and interleukin 8 (IL-8) levels, samples were taken from each volunteer at $t_{0}, t_{1}$, and $t_{2}$ on the treated and untreated scalp sides using standardized scalp wash-ups.

As described above, the hair of the each volunteer was parted in the test area and secured. Prior to each scalp wash-up, 500 $\mu$ l rinse-off buffer containing $100 \times$ Halt ${ }^{\mathrm{TM}}$ Protease Inhibitor, EDTA free (1:100; Thermo Scientific, Rochester, N.Y., USA) were prepared in a 2-ml Eppendorf tube. The rinse-off buffer consisted of $12.49 \mathrm{~g} \mathrm{Na}_{2} \mathrm{HPO}_{4}, 0.63 \mathrm{~g} \mathrm{KH}_{2} \mathrm{PO}_{4}$ (both obtained from Merck, Darmstadt, Germany) and $1 \mathrm{ml}$ Triton X-100 (Sigma, Steinheim, Germany) and 11 demineralized $\mathrm{H}_{2} \mathrm{O}$. The scalp was gently rubbed with constant pressure using a standard cotton bud (Q-tip) moistened with rinse-off buffer. During this procedure, contact with hair fibers was kept to a minimum. The test site (approx. $1.5 \mathrm{~cm}$ ) was rubbed horizontally 20 times followed by multiple elutions of the cotton bud. This procedure was repeated twice and, for storage purposes, cotton buds were then shortened to fit into the tube. For each volunteer, 5 washups were performed. For analysis, samples obtained from the same test sites of the same volunteer at the same point in time were combined. Shortened cotton buds were placed into a cut pipette tip, centrifuged at $14,000 \mathrm{rpm}$ at $4^{\circ} \mathrm{C}$ for $2 \mathrm{~min}$ and the resulting liquid was combined with the corresponding wash-up samples.

Total protein content was analyzed using the Pierce ${ }^{\circledR}$ BCA Protein Assay Kit (Thermo Scientific) according to the manufacturer's instructions. For the determination of IL-1 $\beta$ and IL-1ra levels, commercially available sandwich enzyme-linked immunosorbent assays (ELISAs; Quantikine ${ }^{\circledR}$ Human Immunoassay; R\&D Systems, Wiesbaden, Germany) were utilized according to the manufacturer's protocol. Results were normalized to the individual total protein content of the corresponding sample.

For analysis of IL-8 levels, the ELISA kit Opt EIA ${ }^{\mathrm{TM}}$ Human IL-8 (BD Bioscience, Heidelberg, Germany) was used. The coating buffer was slightly modified and consisted of $4.5 \mathrm{~g} \mathrm{NaHCO}_{3}$ and $1.78 \mathrm{~g} \mathrm{Na}_{2} \mathrm{CO}_{3}$ (both obtained from Merck) and 0.51 demineralized $\mathrm{H}_{2} \mathrm{O}$. All other steps and procedures were carried out as recommended by the manufacturer. 
Self-Assessment Regarding Scalp Condition and Tonic

Efficiency

The volunteers completed self-assessment questionnaires after 2 and 4 weeks, comparing the treated and the untreated scalp side to determine improvements in individual scalp condition (A-D) and agreement with statements regarding tonic efficiency (E-J).

The answers ('I agree/yes', 'I disagree/no') established the agreement with the following statements: (A) 'The condition of my scalp was improved perceivably on the side treated with the test tonic', (B) 'The regular use of the test tonic reduces the dryness of my scalp', (C) 'The regular use of the test tonic diminishes the itching of my scalp', (D) 'The regular use of the test tonic perceivably reduces the tautness of my scalp', (E) 'The test tonic is particularly mild and skin tolerable for my scalp', (F) 'The test tonic is particularly suitable for dry scalp', (G) 'The regular use of the test tonic leaves no residues in the hair', (H) 'I am able to style my hair in the usual way', (I) 'The test tonic provides long-term protection against drying out of my scalp', and (J) 'The test tonic has a calming effect on my scalp'.

For statements B, C and D, volunteers had the additional opportunity to answer with 'I do not have a dry scalp', 'I do not have an itchy scalp' or 'I have no taut sensation on my scalp', respectively.

\section{In vivo Microscopy}

To document changes in scalp condition, in vivo digital microscopy was used at the baseline visit and after 4 weeks of treatment. For this purpose, the volunteer's hair was parted as described above, and 2 photos were taken from both sides of the scalp (untreated/treated). Images were recorded with a VHX-1000 Digital Microscope (16-bit resolution, 54 megapixel, 3 times CCD resolution; Keyence, Neu-Isenburg, Germany).

\section{Statistical Analysis}

A significance level of 0.05 (alpha) was chosen for statistical analysis, based on two-sided hypothesis testing. For analysis, SAS software package for Windows V9.2 and SigmaPlot 11.0 (Systat Software, Inc., Chicago, Ill., USA) were used.

For conductivity measurements, FT-MIR spectroscopy, and the determination of cytokine levels, the results of multiple replications were averaged before analysis. Based on the original data, location parameter median and dispersion parameter interquartile range (IQR) in form of the lower (25\% percentile) and upper quartiles $(75 \%$ percentile) were represented in graphs. Data were checked for normal distribution by means of Shapiro-Wilk test. Due to the fact that the hypothesis of normality was rejected, the Blom-transformed ranks of the original data were used for analysis.

In all statistical models, a subject effect for repeated measures was considered. In order to suitably model intra-individual changes, treatment and point-in-time comparison were performed by incorporating baseline values or values of the untreated control. The initial situation at baseline $t_{0}$ was evaluated by analysis of variance, using 'treatment' as explanatory variable.

The treatment effects were evaluated by analysis of covariance. For the treated versus untreated comparison, 'treatment' and ' $t_{0}$ ' were used as explanatory variables, where 'treatment' was the classification variable and ' $t_{0}$ ' the covariate. For the comparison with baseline, 'point-in-time' and 'untreated' were used as explanatory

Leave-on Tonic for Treating Xerotic, Itchy and Subclinically Inflamed Scalp variables, where 'point-in-time' was the classification variable and 'untreated' the covariate. If applicable, post hoc pairwise comparisons were performed.

Since sample data were not normally distributed in DART-MS analyses, a nonparametric Wilcoxon test for paired samples was performed.

For analysis of self-assessment, absolute and relative frequencies were represented by tables and graphs based on the original data. The relevance of the inquired statements was tested using a binominal test for null hypothesis proportion $\mathrm{p}_{0}=0.5$. Due to rounding errors, the sum of the relative frequencies may deviate from $100 \%$.

\section{Results}

\section{Determination of Scalp Hydration}

The degree of scalp hydration was determined at baseline and after 2 and 4 weeks of treatment to assess the potential of the test tonic to increase scalp moisturization. Starting at comparable baseline levels ( $\mathrm{p}=$ 0.4957 ), at both points in time skin hydration was significantly increased after tonic application in comparison to baseline $\left(t_{1}: p=0.0003 ; t_{2}: p=0.0038 ; n=30\right)$ and untreated control sites $\left(t_{1}: p=0.0002 ; t_{2}: p=0.0117 ; n=\right.$ 30; fig. 1a).

Using a second method for the investigation of scalp moisturization, FT-MIR measurements analyzing the ratio of intensities of amide I and II bands in the corresponding spectra were performed at $t_{0}, t_{1}$, and $t_{2}$ (fig. $1 b$ ). Starting at similar baseline levels $(\mathrm{p}=0.5746)$, the ratio of amide bands (I/II) in treated sites was significantly increased $\left(\mathrm{t}_{1}: \mathrm{p}=0.0003 ; \mathrm{t}_{2}: \mathrm{p}=0.0465 ; \mathrm{n}=30\right)$ compared to baseline.

\section{Scalp Substantivity of Urea and Lactate Using DART- MS Analysis}

To gain more information on the substantivity of urea and lactate in the course of tonic treatment, DARTMS analysis was performed $(n=29)$. Following adjustment of values to basement levels (urea: $p=0.7564$; lactate: $p=0.1989$ ), the relative amount of urea was significantly increased after 2 and 4 weeks of tonic application in comparison to baseline $\left(t_{1}: p=0.0010 ; t_{2}\right.$ : $\mathrm{p}=0.0027)$ and untreated control sites $\left(\mathrm{t}_{1}: \mathrm{p}=0.0002 ; \mathrm{t}_{2}\right.$ : $\mathrm{p}=0.0033$; fig. $2 \mathrm{a}$ ). For the tonic-treated sites, a significant decrease of urea was shown with respect to the results obtained after 2 and 4 weeks of treatment $\left(t_{1} / t_{2}: p=\right.$ 0.0187 ). Figure $2 \mathrm{~b}$ illustrates the significant augmentation in the relative amount of lactate comparing the tonictreated to the untreated scalp site $\left(\mathrm{t}_{1}: \mathrm{p}=0.0007 ; \mathrm{t}_{2}: \mathrm{p}=\right.$ $0.0321)$ after 2 and 4 weeks of treatment as well as an 


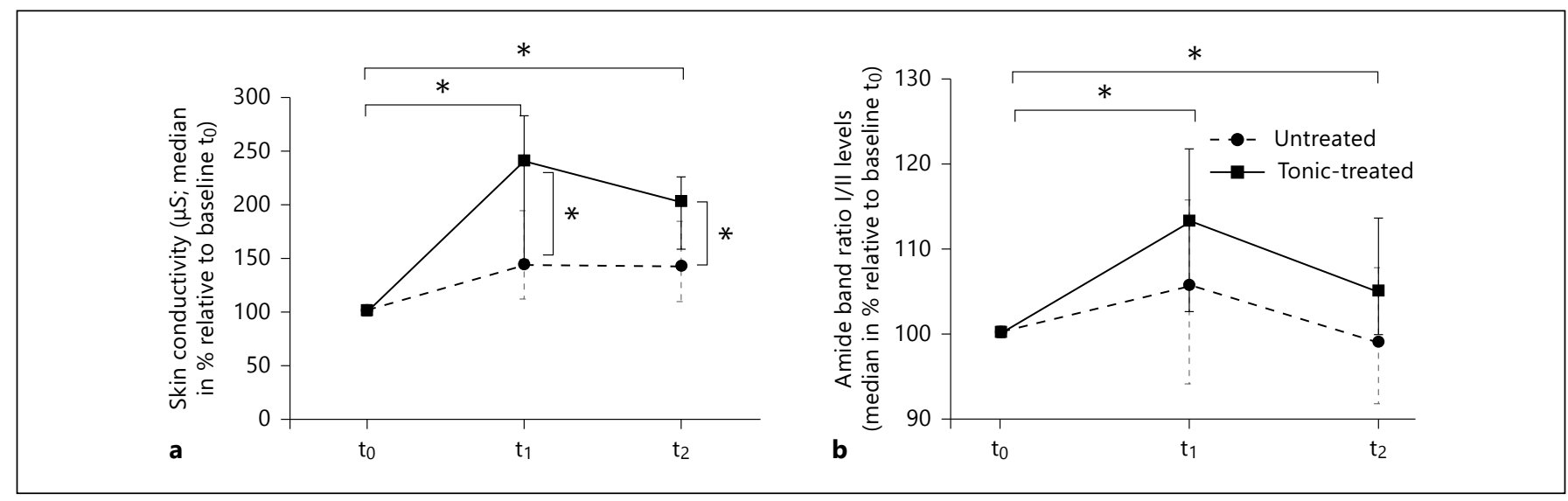

Fig. 1. Increase in scalp hydration after 2 and 4 weeks of tonic application. a Corneometry. Results are shown as median in \% relative to baseline $t_{0}(n=30)$. Depicted is the $25 / 75$ th percentile for the untreated (gray, dashed line) and treated (black, solid line) sites. b FT-MIR spectroscopy analyzing the amide band ratio I/II.
Results are shown as median in \% relative to baseline $t_{0}(n=30)$. Depicted is the 25/75th percentile for the untreated (gray, dashed line) and treated (black, solid line) sites. Significant differences are marked with an asterisk $\left({ }^{*} \mathrm{p} \leq 0.05\right)$.

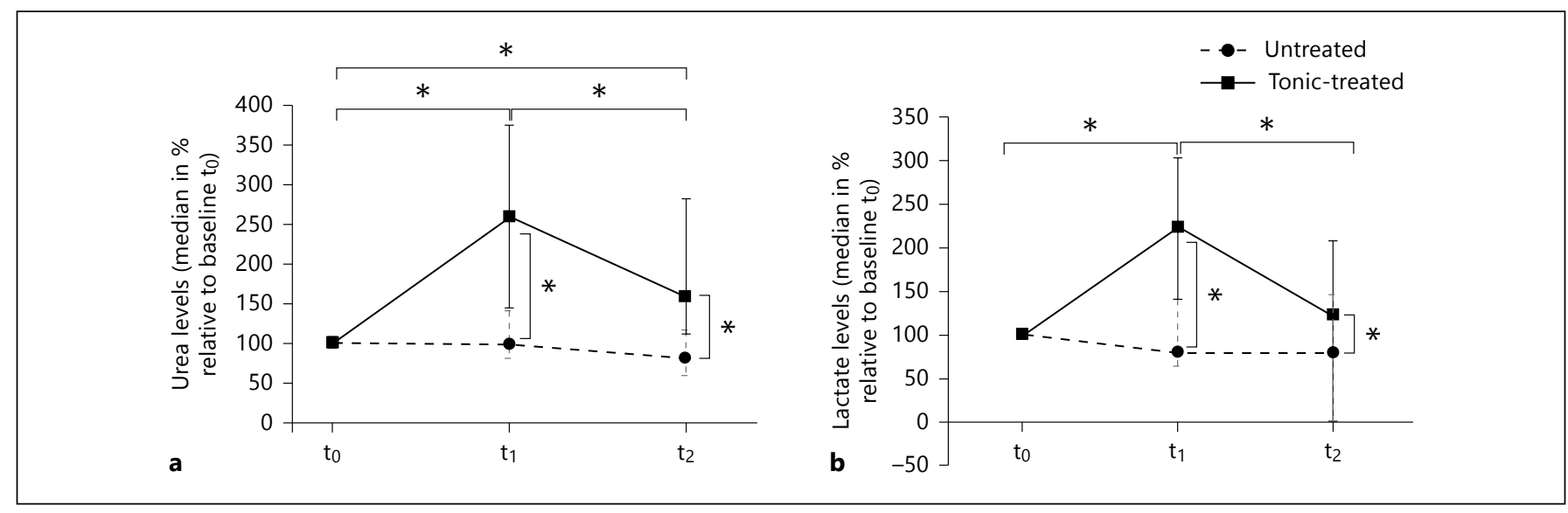

Fig. 2. Substantivity of urea and lactate after 2 and 4 weeks of tonic application using DART-MS analysis. a Urea levels. b Lactate levels. Results are shown as median in \% relative to baseline $t_{0}$ $(n=29)$. Depicted is the $25 / 75$ th percentile for the untreated (gray, dashed line) and treated (black, solid line) sites. Significant differences are marked with an asterisk $(* \mathrm{p} \leq 0.05)$. increase in lactate after 2 weeks of treatment in comparison to baseline $\left(t_{1}: p=0.0009\right)$. Comparing tonic-treated sites, data obtained after 2 and 4 weeks of treatment showed a significant reduction in the relative amount of lactate $\left(\mathrm{t}_{1} / \mathrm{t}_{2}: \mathrm{p}=0.009\right)$.

\section{Determination of Free Fatty Acids and Triglycerides Using FT-MIR}

Using FT-MIR ( $\mathrm{n}=30$ ), levels of triglycerides, free fatty acids, and total lipids were detected at comparable baseline levels on both scalp sides (triglyceride: $p=0.5168$, free fatty acids: $p=0.2040$, total lipids: $p=0.5513$ ). Significantly increased triglyceride levels were found in the treated sites both in comparison to baseline $\left(\mathrm{t}_{1}: \mathrm{p}=0.0072\right.$; $\left.t_{2}: p=0.0105\right)$ and control $\left(t_{1}: p=0.0038 ; t_{2}: p=0.0005\right.$; fig. 3a). In the course of the study, the amount of free fatty acids was significantly reduced in the tonic-treated scalp site compared to baseline $\left(\mathrm{t}_{1}: \mathrm{p}=0.0000 ; \mathrm{t}_{2}: \mathrm{p}=\right.$ $0.0000)$ and control $\left(t_{1}: p=0.0000 ; t_{2}: p=0.0001\right.$; fig. $\left.3 b\right)$. As demonstrated in figure $3 c$, the use of the tonic affected 
neither the amount of total lipids compared to baseline $\left(\mathrm{t}_{1}: \mathrm{p}=0.0970 ; \mathrm{t}_{2}: \mathrm{p}=0.2841\right)$ nor the control $\left(\mathrm{t}_{1}: \mathrm{p}=\right.$ $\left.0.4659 ; \mathrm{t}_{2}: \mathrm{p}=0.3109\right)$.

\section{Determination of Scalp Cytokine Levels}

To study effects of the tonic on the IL- $1 \mathrm{ra} / \mathrm{IL}-1 \beta$ ratios as well as IL-8 levels, samples were taken from each volunteer at $\mathrm{t}_{0}, \mathrm{t}_{1}$, and $\mathrm{t}_{2}$ on treated and untreated scalp sides $(\mathrm{n}=30)$. As shown in figure $4 \mathrm{a}$, a significant reduction in the cytokine ratio IL-1 ra/IL-1 $\beta$ was achieved after 2 and 4 weeks of tonic treatment regarding the treated in comparison to the untreated site $\left(\mathrm{t}_{1}: \mathrm{p}=0.0376 ; \mathrm{t}_{2}: \mathrm{p}=0.0479\right)$ and after 2 weeks comparing the treated site to baseline $(\mathrm{p}=0.0112)$. Figure $4 \mathrm{~b}$ shows the decrease in pro-inflammatory cytokine IL- 8 levels after 2 and 4 weeks of treatment with the test tonic. Following exclusion of all volunteers not displaying a detectable IL-8 expression at baseline, a significant reduction in IL-8 levels compared to baseline was detected after 2 weeks of treatment ( $\mathrm{p}=$ $0.0204 ; \mathrm{n}=28$ ).

\section{Self-Assessment Regarding Scalp Condition and Tonic Efficiency}

In order to elucidate any changes in the volunteer's perception of the dry and itchy scalp condition as well as to determine the degree of tonic efficiency, volunteers carried out a self-assessment after 2 and 4 weeks of treatment (fig. 5a, b). Statements A to J were confirmed with statistical evidence ( $\mathrm{p} \leq 0.0433$ for A-D; $\mathrm{p} \leq 0.0001$ for $\mathrm{E}-\mathrm{J}$ ) at both points in time. The exception was statement A, which showed no significance after two weeks of use. A large majority of volunteers $(>80 \%)$ 'agreed' with statements E-J.

Some volunteers did not show the phenotype required to comment on statements B, C and D and were, for this reason, not taken into account for statistical analysis. Also, variations in the number of answers $(n=20-30)$ are due to implausible answers given by a few volunteers during the study. These answers had to be excluded from analysis.

Results demonstrated that the tonic formulation was very well tolerated by the study population over the entire period of usage, since no incompatibility reactions were observed. Results also showed that the tonic allowed excellent hair management.

\section{In vivo Microscopy}

An example of the improvement in scalp condition achieved by the tonic treatment is presented in figure 6 , showing an untreated and a tonic-treated scalp site after 4 weeks.

Leave-on Tonic for Treating Xerotic, Itchy and Subclinically Inflamed Scalp

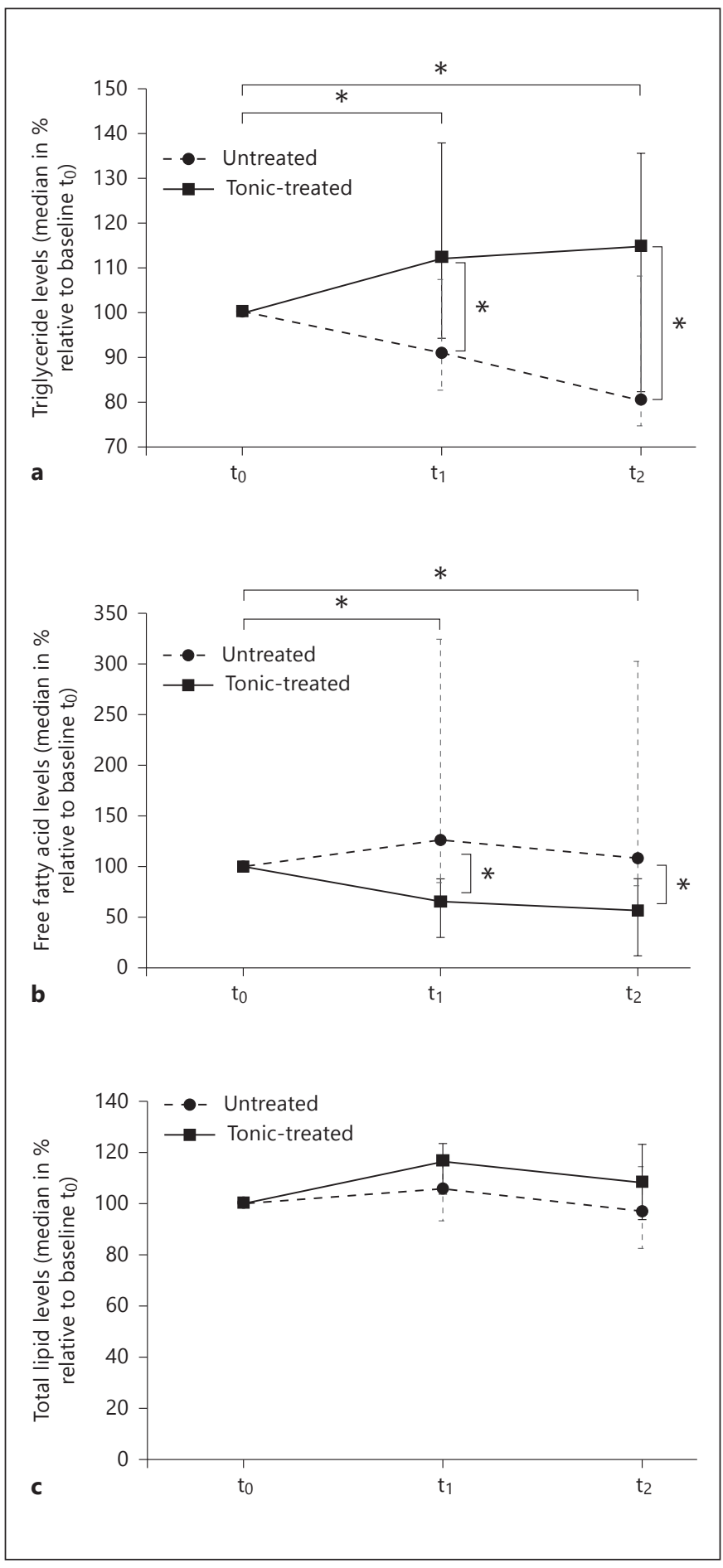

Fig. 3. Determination of triglyceride, free fatty acid, and total lipid levels after 2 and 4 weeks of tonic application using FT-MIR. a Alterations in triglyceride levels. b Changes in free fatty acid levels. c Changes in the amount of total lipids. Results are shown as median in $\%$ to relative baseline $t_{0}(n=30)$. Depicted is the 25/75th percentile for the untreated (gray, dashed line) and treated (black, solid line) sites. Significant differences are marked with an asterisk ( $\left.{ }^{*} \mathrm{p} \leq 0.05\right)$.

Skin Pharmacol Physiol 2013;26:108-118 


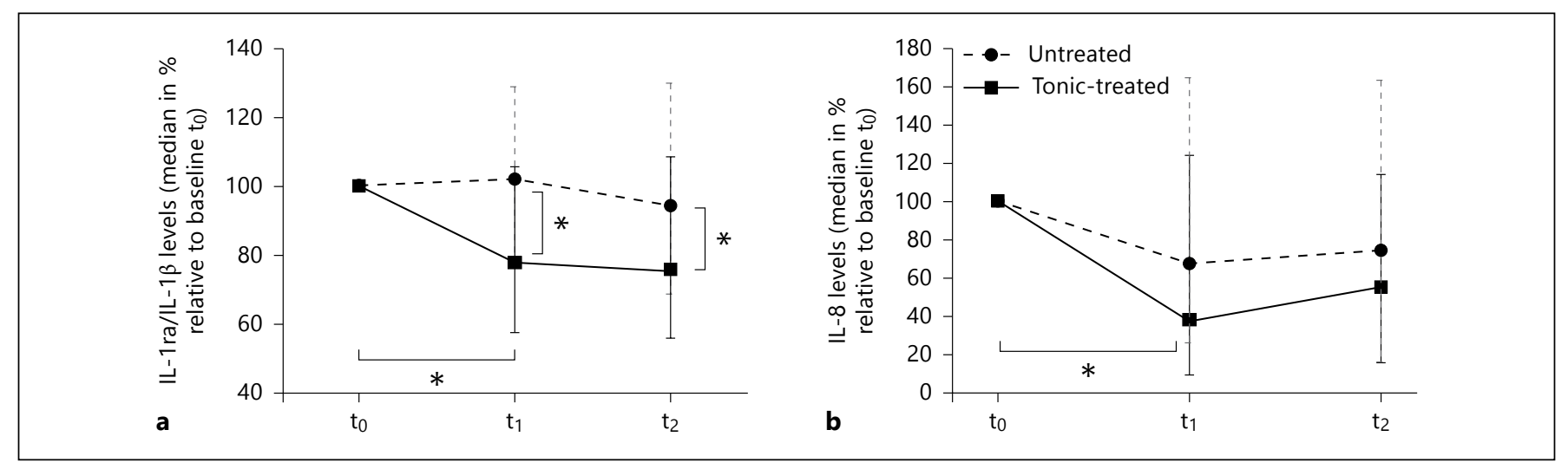

Fig. 4. Levels of pro-inflammatory markers after 2 and 4 weeks of tonic application. a IL-1ra/IL-1 $\beta$ levels $(n=30)$. b IL-8 levels $(\mathrm{n}=28$, all volunteers with detectable IL-8 levels). Results are shown as median in \% relative to baseline $t_{0}$. Depicted is the 25/75th percentile for the untreated (gray, dashed line) and treated (black, solid line) sites. Significant differences are marked with an asterisk $(* \mathrm{p} \leq 0.05)$.

\section{Discussion}

The status of scalp skin is affected by endogenous parameters, such as NMF levels, the condition of the skin barrier, sebum content, and age. At the same time, it is influenced by exogenous factors, such as microbiological flora, environmental stress, as well as hair and skin care.

Dry and itchy skin conditions may result from an impaired SC function $[5,22]$. Even minor defects of the SC barrier may lead to superficial dehydration and the secretion of pro-inflammatory cytokines, which cause cutaneous (micro)inflammation [23]. This process can induce pruritus, which leads to scratching, subsequently resulting in skin barrier damage and the exacerbation of xerotic scalp conditions.

The overall objective of this study was to test the hypothesis that a leave-on tonic containing the anti-inflammatory flavonoid licochalcone $\mathrm{A}$ in combination with the NFMs urea and lactate and the local anesthetic polidocanol (1) hydrates the scalp, (2) normalizes lipid levels in the SC, (3) reduces microinflammation, and (4) improves scalp pruritus and dryness.

To address our hypothesis, we performed an in vivo split head design study and investigated the effect of the new leave-on tonic on scalp hydration. We utilized inductive corneometry and FT-MIR to noninvasively and repetitively study the hydration status of the scalp on the same skin area at different points in time. Corneometry results confirmed that, after 2 and 4 weeks of tonic treatment, a significant increase in skin hydration was detected both in comparison to baseline and the untreated con- trol site. The above findings of improved scalp moisturization were further supported by FT-MIR measurements, when comparing the ratio of intensities of amide I/II bands in the MIR spectrum.

We next investigated if the improved scalp moisturization after tonic application is accompanied by alterations in urea and lactate levels. Reduced NMF levels have been implicated in dry skin conditions [6], and lactate levels have been shown to correlate significantly with the hydration status in the SC [24]. Using DART-MS and comparing the treated to the untreated scalp, a significant increase in the relative amount of urea and lactate was detected. To exclude any direct effects by the tonic ingredients, the hair was shampooed $48 \mathrm{~h}$ prior to measurements and no tonic was applied. When these prerequisites are taken into consideration, our data point to a high degree of tonic substantivity.

For skin conductivity a slight decrease and for the amount of urea and lactate significant decreases after 4 weeks of treatment compared to 2 weeks tonic treatment were detected. This observation might be based on the fact that the scalp skin is becoming accustomed to the application of the leave-on tonic and might also be due to data fluctuations during measurements. However, a significant increase in skin conductivity and in urea and lactate levels comparing treated to untreated scalp sites was still determined after 4 weeks of treatment for all 3 parameters. Moreover, the volunteers perceived still additional improvements in general scalp condition as well as scalp dryness or itching after 4 weeks of treatment when comparing the self-assessment data to the 2 -week results. 


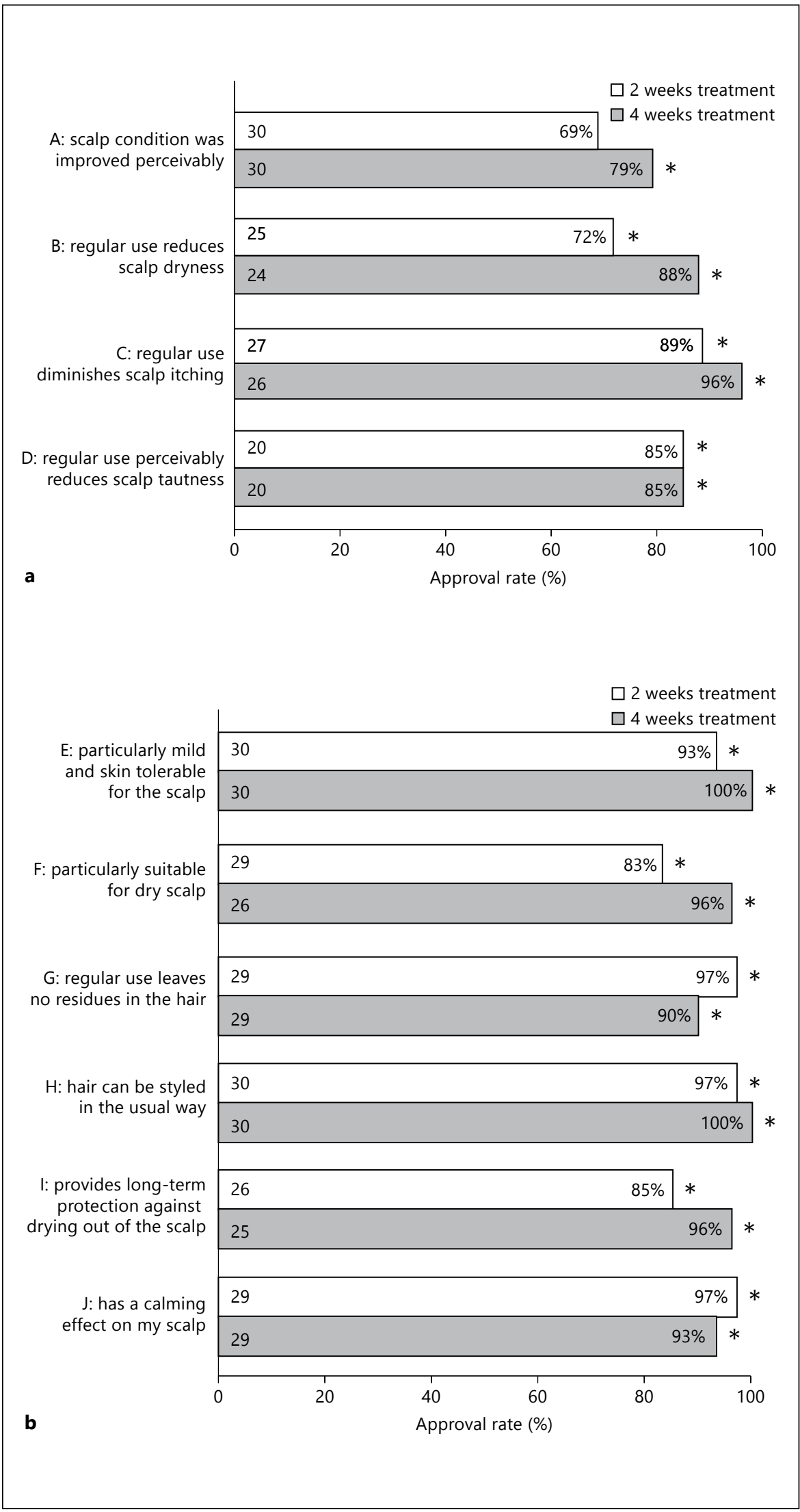

Fig. 5. Self-assessment of volunteers after 2 and 4 weeks of tonic application with respect to a improvements in scalp condition $(\mathrm{n}=20-30)$ and $\mathbf{b}$ tonic efficacy $(\mathrm{n}=25-$ 30). Shown is the approval rate (\%) for each statement. Significant differences are marked with an asterisk $\left({ }^{*} \mathrm{p} \leq 0.05\right)$.

Leave-on Tonic for Treating Xerotic, Itchy and Subclinically Inflamed Scalp 
Fig. 6. In vivo microscopy images obtained from a representative volunteer show the improvement in scalp condition achieved after a 4-week tonic treatment. Shown are the untreated scalp site (left) and the tonictreated site (right).
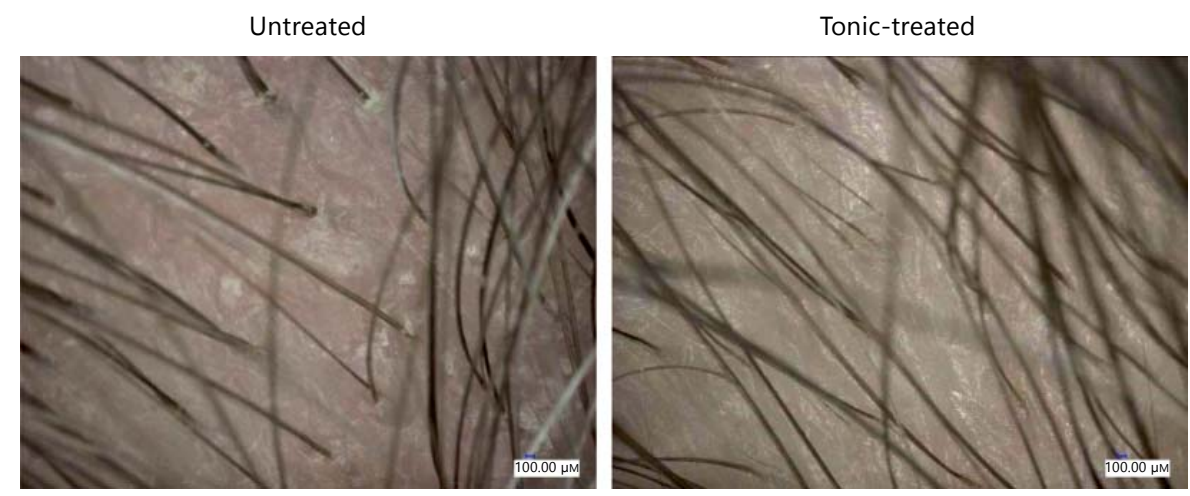

The function of the cutaneous lipid barrier is dependent on its structural components, such as SC lipids and NMFs [25]. To address our second objective, we next focused on the determination of the amount of free fatty acids and triglycerides. Triglycerides on the scalp are partially hydrolyzed by lipases, which are generated by lipase-producing microorganisms of human skin flora [26]. Triglyceride hydrolysis by the epidermal acid lipase, which is delivered to the SC via the lamellar granules, is also discussed in the literature [27]. However, the significance of microbial triglyceride hydrolysis compared with acid lipase hydrolysis remains to be further investigated. In addition, the continuous removal of the uppermost skin layer releases free fatty acids [28], and the activity of fungal species such as Malassezia has also been shown to decrease the amount of triglycerides [29]. An involvement of cutaneous microorganisms has been implicated in inflammatory diseases such as acne, psoriasis, and seborrheic dermatitis [30].

Aberrant lipids levels have been linked to conditions such as atopic dermatitis and lamellar ichthyosis [31,32]. In surfactant-induced dry scaly skin, alterations in lipid composition have been shown [33]. Also, certain free unsaturated fatty acids have proven to be highly irritating to the skin, being able to induce inflammation and augmentation of IL- $1 \alpha$ and IL-8 levels, as well as influence keratinocyte differentiation in the epidermis $[5,34]$.

Importantly, our data showed that the amount of total lipids was unchanged. This result is of special relevance, since the deposition of extra lipids might give the scalp an undesired oily or greasy appearance. In parallel, we observed a significant increase in triglyceride and a significant reduction in free fatty acid levels in the tonic-treated scalp site compared to baseline and control. In this context, these data indicate beneficial tonic-induced effects on the dry scalp.
It is tempting to speculate that the change in lipid composition observed in our study might be due to a modified activity of cutaneous microflora, namely Malassezia spp. A recent study showed positive effects of a formulation containing urea and lactic acid plus propylene glycol in seborrheic dermatitis, a scalp condition commonly associated with Malassezia spp. [35].

However, as shown above, the formation of free fatty acids can be of varying origin. To investigate microbial scalp colonization and activity in more detail, an additional study to determine the glyceride class compounds prior to cleavage [28] might provide more information. Although the test tonic did not contain any common antimycotic actives, the formulation might exert antimicrobial effects, preventing triglyceride degradation possibly mediated by lactate, which has been linked to antimicrobial characteristics $[36,37]$.

Dry skin has been associated with an impaired barrier function and can release pro-inflammatory cytokines [6]. To evaluate the third part of our hypothesis, we investigated if application of the test tonic causes alterations in cytokine levels. The IL-1 system plays a central part in skin physiology. By competitively binding to the IL-1 receptor type I (IL-1RI), the anti-inflammatory IL-1 ra prevents the binding of IL- $1 \alpha$ and IL- $1 \beta$. An increased IL$1 \mathrm{ra} / \mathrm{IL}-1 \alpha$ ratio is a marker of skin inflammations of different origins [38]. Since IL-1ra can also prevent binding of IL- $1 \beta$ to the IL-1RI, the ratio of IL-1ra/IL- $1 \beta$ can as well be seen as a marker of inflammation [39].

Analysis of the IL-1 ra/IL- $1 \beta$ ratio in scalp wash-ups showed that a significant reduction was achieved after 2 and 4 weeks of tonic treatment, indicating a decrease in inflammatory processes. We next determined the levels of the pro-inflammatory cytokine IL-8. IL-8 represents a CXC chemokine with strong chemotactic properties and is, like IL-1 $\beta$, expressed in keratinocytes. High levels of 
IL- 8 have been associated with active inflammatory processes [40] and increased IL-8 levels could be linked to inflammatory scalp diseases, such as dandruff, seborrheic dermatitis, and psoriasis [41]. Also, in patients with atopic dermatitis, SC IL-8 levels in lesions were significantly higher than in healthy controls [42]. Xerotic scalp conditions can lead to scratching-induced superficial wounds. In this context, it is interesting to note that epidermal injury is a major inducer of IL- 8 expression in skin wounds [43]. The expression of IL- 8 is further regulated by IL- $1 \beta$ [44]. After 2 weeks of tonic treatment, our results showed a significant reduction in IL-8 levels compared to baseline.

Taken together, the significant reduction in the IL-1ra/ IL- $1 \beta$ ratio and also the significant decrease in IL- 8 levels after 2 weeks of treatment support the assumption that the new tonic exerts anti-inflammatory effects.

To verify our fourth objective, we investigated if these results translate into in vivo effects. Volunteers completed self-assessment questionnaires focusing on scalp condition and tonic efficiency after regular tonic application.

After 4 weeks of use, comparing the treated to the untreated scalp site, volunteers reported a significant subjective amelioration of symptoms, such as scalp dryness, pruritus, and tautness. These results were confirmed by the in vivo microscopy, comparing the untreated to the treated scalp side. The overall scalp condition was considered to have improved significantly on the treated side. The volunteers stated that they were able to style their hair in the usual way, showing that the tonic not only improves scalp condition, but also imparts a good cosmetic appearance to the hair.

In conclusion, our results demonstrate that application of the new leave-on tonic containing urea, lactate, polidocanol, and licochalcone A resulted in a significant increase in scalp hydration, a normalization of lipid alterations and a significant reduction in key pro-inflammatory markers. Our data provide evidence for the test tonic being a formulation which significantly reduces the symptoms of a dry, itchy, and subclinically inflamed scalp, while allowing excellent hair management.

\section{Acknowledgements}

We kindly thank Dr. Janina Lorenz for the development of the IL-8 assay. We further thank Mrs. Claudia Gillner, Mrs. Danijela Strazivuk, Mrs. Brit Kayser, and Mrs. Manuela Lütgens (hair studio, Beiersdorf test center) for conducting hair washes and tonic application during the prescreening and the in vivo study. We also thank Mr. Bernie Larisch for the critical review of the manuscript.

\section{References}

$>1$ Elewski BE: Clinical diagnosis of common scalp disorders. J Investig Dermatol Symp Proc 2005;10:190-193.

$>2$ Misery L, Sibaud V, Ambronati M, Macy G, Boussetta S, Taieb C: Sensitive scalp: does this condition exist? An epidemiological study. Contact Dermatitis 2008;58:234-238.

$\checkmark 3$ Loden M: Role of topical emollients and moisturizers in the treatment of dry skin barrier disorders. Am J Clin Dermatol 2003;4: 771-788.

4 Saint-Martory C, Roguedas-Contios AM, Sibaud V, Degouy A, Schmitt AM, Misery L: Sensitive skin is not limited to the face. Br J Dermatol 2008;158:130-133.

5 Bin Saif GA, Ericson ME, Yosipovitch G: The itchy scalp-scratching for an explanation. Exp Dermatol 2011;20:959-968.

$\checkmark 6$ Rawlings AV, Matts PJ: Stratum corneum moisturization at the molecular level: an update in relation to the dry skin cycle. J Invest Dermatol 2005;124:1099-1110.

-7 Sugawara T, Kikuchi K, Tagami H, Aiba S, Sakai S: Decreased lactate and potassium levels in natural moisturizing factor from the stratum corneum of mild atopic dermatitis patients are involved with the reduced hydration state. J Dermatol Sci 2012;66:154-159.
>8 Freitag G, Höppner T: Results of a postmarketing drug monitoring survey with a polidocanol-urea preparation for dry, itching skin. Curr Med Res Opin 1997;13:529-537.

$\checkmark 9$ Leopold CS, Maibach HI: Effect of cutaneously applied nonionic surfactants and local anesthetic bases on thermal sensations. Pharmazie 2004;59:50-54.

10 Saint-Léger D, François AM, Lévêque JL, Stoudemayer TJ, Kligman AM, Grove G: Stratum corneum lipids in skin xerosis. Dermatologica 1989;178:151-155.

11 Kim KS, Shin MK, Kim JH, Kim MH, Haw CR, Park HK: Effects of atopic dermatitis on the morphology and water content of scalp hair. Microsc Res Tech 2012;75:620-625.

12 Shibata S: A drug over the millennia: pharmacognosy, chemistry, and pharmacology of licorice. Yakugaku Zasshi 2000;120:849-862.

13 Fiore C, Eisenhut M, Ragazzi E, Zanchin G, Armanini D: A history of the therapeutic use of liquorice in Europe. J Ethnopharmacol 2005;99:317-324.

14 Friis-Moller A, Chen M, Fuursted K, Christensen SB, Kharazmi A: In vitro antimycobacterial and antilegionella activity of licochalcone A from chinese licorice roots. Planta Med 2002;68:416-419.
15 Funakoshi-Tago M, Tanabe S, Tago K, Itoh H, Mashino T, Sonoda Y, Kasahara T: Licochalcone A potently inhibits tumor necrosis factor alpha-induced nuclear factor-kappaB activation through the direct inhibition of IkappaB kinase complex activation. Mol Pharmacol 2009;76:745-753.

16 Furuhashi I, Iwata S, Shibata S, Sato T, Inoue $\mathrm{H}$ : Inhibition by licochalcone A, a novel flavonoid isolated from liquorice root, of IL1beta-induced PGE2 production in human skin fibroblasts. J Pharm Pharmacol 2005;57: 1661-1666

17 Kolbe L, Immeyer J, Batzer J, Wensorra U, tom Dieck K, Mundt C, Wolber R, Stäb F, Schönrock U, Ceilley RI, Wenck H: Anti-inflammatory efficacy of Licochalcone A: correlation of clinical potency and in vitro effects. Arch Dermatol Res 2006;298:23-30.

18 Weber TM, Ceilley RI, Buerger A, Kolbe L, Trookman NS, Rizer RL, Schoelermann A: Skin tolerance, efficacy, and quality of life of patients with red facial skin using a skin care regimen containing Licochalcone A. J Cosmet Dermatol 2006;5:227-232.
Leave-on Tonic for Treating Xerotic, Itchy and Subclinically Inflamed Scalp 
19 Udompataikul M, Srisatwaja W: Comparative trial of moisturizer containing licochalcone $\mathrm{A}$ vs. hydrocortisone lotion in the treatment of childhood atopic dermatitis: a pilot study. J Eur Acad Dermatol Venereol 2011;25:660 665.

20 Triebskorn A, Gloor M, Greiner F: Comparative investigations on the water content of the stratum corneum using different methods of measurement. Dermatologica 1983;167:6469.

21 Mess A, Enthaler B, Fischer M, Rapp C, Pruns JK, Vietzke J-P: A novel sampling method for identification of endogenous skin surface compounds by use of DART-MS and MALDI-MS. Talanta 2013;103:398-402.

-22 Rawlings AV, Harding CR: Moisturization and skin barrier function. Dermatol Ther 2004;17(suppl 1):43-48.

-23 Wood LC, Jackson SM, Elias PM, Grunfeld C, Feingold KR: Cutaneous barrier perturbation stimulates cytokine production in the epidermis of mice. J Clin Invest 1992;90:482-487.

-24 Nakagawa N, Sakai S, Matsumoto M, Yamada K, Nagano M, Yuki T, Sumida Y, Uchiwa H: Relationship between NMF (lactate and potassium) content and the physical properties of the stratum corneum in healthy subjects. J Invest Dermatol 2004;122:755-763.

25 Del Rosso JQ, Levin J: The clinical relevance of maintaining the functional integrity of the stratum corneum in both healthy and diseaseaffected skin. J Clin Aesthet Dermatol 2011;4: 22-42.

26 Saunders CW, Scheynius A, Heitman J: Malassezia fungi are specialized to live on skin and associated with dandruff, eczema, and other skin diseases. PLoS Pathog 2012;8:e1002701.

-27 Drake DR, Brogden KA, Dawson DV, Wertz PW: Thematic review series: skin lipids. Antimicrobial lipids at the skin surface. J Lipid Res 2008;49:4-11.
28 Michael-Jubeli R, Bleton J, Baillet-Guffroy A: High-temperature gas chromatography-mass spectrometry for skin surface lipids profiling. J Lipid Res 2011;52:143-151.

29 Ro BI, Dawson TL: The role of sebaceous gland activity and scalp microfloral metabolism in the etiology of seborrheic dermatitis and dandruff. J Investig Dermatol Symp Proc 2005; 10:194-197.

30 Smith KR, Thiboutot DM: Thematic review series: skin lipids. Sebaceous gland lipids: friend or foe? J Lipid Res 2008;49:271-281.

31 Fölster-Holst R, Dähnhardt-Pfeiffer S, Dähnhardt D, Proksch E: The role of skin barrier function in atopic dermatitis: an update. Expert Rev Dermatol 2012;7:247-257.

32 Elias PM, Williams ML, Feingold KR: Abnormal barrier function in the pathogenesis of ichthyosis: therapeutic implications for lipid metabolic disorders. Clin Dermatol 2012;30: 311-322.

33 Imokawa G: Surfactant-induced depletion of ceramides and other intercellular lipids: implication for the mechanism leading to dehydration of the stratum corneum. Exog Dermatol 2004;3:81-98.

34 Katsuta Y, Iida T, Inomata S, Denda M: Unsaturated fatty acids induce calcium influx into keratinocytes and cause abnormal differentiation of epidermis. J Invest Dermatol 2005; 124:1008-1013.

35 Emtestam L, Svensson A, Rensfeldt K: Treatment of seborrhoeic dermatitis of the scalp with a topical solution of urea, lactic acid, and propylene glycol (K301): results of two double-blind, randomised, placebo-controlled studies. Mycoses 2012;55:393-403.

36 Ostling CE, Lindgren SE: Inhibition of enterobacteria and Listeria growth by lactic, acetic and formic acids. J Appl Bacteriol 1993;75: $18-24$.
37 Sallam KI: Antimicrobial and antioxidant effects of sodium acetate, sodium lactate, and sodium citrate in refrigerated sliced salmon. Food Control 2007; 18:566-575.

38 Terui T, Hirao T, Sato Y, Uesugi T, Honda M, Iguchi M, Matsumura N, Kudoh K, Aiba S, Tagami H: An increased ratio of interleukin-1 receptor antagonist to interleukin-1alpha in inflammatory skin diseases. Exp Dermatol 1998;7:327-334.

39 Jensen LE: Targeting the IL-1 family members in skin inflammation. Curr Opin Investig Drugs 2010;11:1211-1220.

40 Arican O, Aral M, Sasmaz S, Ciragil P: Serum levels of TNF-alpha, IFN-gamma, IL-6, IL-8, IL-12, IL-17, and IL-18 in patients with active psoriasis and correlation with disease severity. Mediators Inflamm 2005;2005:273279.

- 41 Perkins MA, Cardin CW, Osterhues MA, Robinson MK: A non-invasive tape absorption method for recovery of inflammatory mediators to differentiate normal from compromised scalp conditions. Skin Res Technol 2002;8:187-193.

-42 Amarbayasgalan T, Takahashi H, Dekio I, Morita E: Interleukin-8 content in the stratum corneum as an indicator of the severity of inflammation in the lesions of atopic dermatitis. Int Arch Allergy Immunol 2012;160:6374.

43 Roupé KM, Nybo M, Sjöbring U, Alberius P, Schmidtchen A, Sørensen OE: Injury is a major inducer of epidermal innate immune responses during wound healing. J Invest Dermatol 2010;130:1167-1177.

44 Myokai F, Koyama E, Nishikawa K, Noji S, Murayama Y, Taniguchi S: Aspects of interleukin- 8 gene expression by gingival and dermal fibroblasts stimulated with interleukinlbeta or tumour necrosis factor alpha. J Int Acad Periodontol 2004;6:21-28. 